\title{
Geometric Optimization in Presence of Contact Singularities
}

\author{
Jungsun Park* and W. J. Anderson ${ }^{\dagger}$ \\ University of Michigan, Ann Arbor, Michigan 48109
}

\begin{abstract}
The stress singularity of a sharp wedge contacting a frictionless half plane can be avoided by changing the wedge shape. Shape optimization is accomplished with the geometric strain method (GSM), an optimality criterion method. Several numerical examples are provided for different materials in the wedge and half plane to avoid stress singularity near the sharp corner of the wedge. Optimum wedge shapes are obtained and critical corner angles are compared with the angles from analytical contact mechanics. Numerical results are well matched to analytical and experimental results. It is shown that shape optimization by GSM is a useful tool to reshape the wedge and to avoid a stress singularity. The method applies to more general geometries where the singular behavior would be difficult to avoid by classical means.
\end{abstract}

$\mathcal{D}$

$[D]$

$[E]$

$f$

$[K]$

$\left[N_{i}\right]$

$N E$

$n_{e}$

$\boldsymbol{P}$

$\boldsymbol{P}_{G}$

$p$

$\operatorname{Re}(\cdot)$

$r, \theta$

[T]

$\boldsymbol{u}$

$\boldsymbol{u}_{G}$

$u_{r}, u_{\theta}$

$\boldsymbol{x}$

$x_{d}$

$\alpha, \beta$

$\alpha_{f}$

$\Gamma_{d}, \Gamma_{i}, \Gamma_{t}, \Gamma_{u}$

$\gamma$

$\gamma_{s}$

$\Delta \ell_{1}, \Delta \ell_{2}$

$\epsilon_{r r}, \epsilon_{r \theta}, \epsilon_{\theta \theta}$

$\epsilon_{G x}, \epsilon_{G y}, \epsilon_{G x y}$

$\mu$

$\sigma_{r r}, \sigma_{r \theta}, \sigma_{\theta \theta}$

$\sigma_{1}, \sigma_{2}, \sigma_{3}, \sigma_{o 1}, \sigma_{o 2}$

$\phi$

$\Omega_{b}, \Omega_{f}$

Subscript

G

$=$ geometric

and 2

\section{Superscripts}

(e)

I, II

$=$ element $e$

$=$ half plane and wedge

\section{Introduction}

$=$ shear modulus

= global stiffness matrix

$=$ displacement shape function

$=$ total number of elements

$=$ total number of nodes in an element

$=$ applied load vector

$=$ equivalent load due to geometric strain

= order of stress singularity

= real part of a complex

$=$ polar coordinates

$=$ coordinate transformation matrix

$=$ displacement

$=$ geometric displacement

$=$ displacement components

$=$ coordinate vector

$=$ design variable

$=$ Dundurs parameters

$=$ acceleration factor

$=$ exterior design, interior, traction, and constrained boundary

$=$ wedge angle

$=$ critical wedge angle separating between presence and absence of singularity

$=$ length in infinitesimal element

$=$ strain components

= geometric strain components

$=$ coefficient of friction

$=$ Poisson's ratio

$=$ stress components

= objective stress in principal axes 1

$=$ Airy stress function

$=$ applied body force domain and fully stressed domain publication Sept. 15, 1994. Copyright (C) 1994 by the American Institute of Aeronautics and Astronautics, Inc. All rights reserved.

*Visiting Scientist, Aerospace Engineering. Member AIAA.

† Professor, Aerospace Engineering. Senior Member AIAA.
HE stress distribution in an infinite single wedge was solved by Flamant using the three-dimensional Boussinesq solution ${ }^{1,2}$ (the Flamant solution is sometimes called simple radial distribution). Michell $^{2,3}$ extended the theory of two-dimensional wedge contact for loads on the wedge faces or for tractions on the infinite boundary by using the Boussinesq and Flamant solution.

Williams ${ }^{4}$ developed the stress distributions near a singularity for various boundary conditions for angular corners of plates in extension. Bogy ${ }^{5,6}$ studied the singular stress field for two-wedge bonded contact by using the Mellin transform ${ }^{7}$ and William's method.

A wedge contacting a half plane has been studied by Dundurs and $\mathrm{Lee}^{8}$ to investigate the stress singularity near a sharp edge for frictionless contact. Gdoutos and Theocaris ${ }^{9}$ extended the problem for frictional and bonded contact by using a simplified approach. Based on the previous work, Comninou ${ }^{10}$ found critical wedge angles at which power stress singularities disappear for frictional contact.

Historically, notches have been used to eliminate high stresses near disks shrunk fit on circular shafts. ${ }^{11}$ Experiments have been done to relieve the stress singularity at the re-entrant sharp corner using notches (Bijak-Zochowski et al. ${ }^{12}$ ).

Benedict and Taylor ${ }^{13,14}$ used the Lagrangian multiplier technique to transform the inequality equilibrium and optimized several contact problems by a direct minimization of the potential energy function. Taylor ${ }^{15}$ presented a relation to design the maximum value of contact pressure.

Recently, a few studies ${ }^{16-18}$ have been done on the mathematical theory of shape optimization for contact problems on the basis of optimization theory. The mathematical theories are limited to a simple problem such as the Signorini problem, ${ }^{19}$ in which a plane elastic body is supported by a rigid frictionless foundation. The theory is not robust because the objective functional is not continuously differentiable.

The present study starts from the idea that the stress singularity of the sharp wedge contacting the half plane may disappear by changing the wedge shape using the geometric strain method, ${ }^{20,21}$ an optimality criterion method. Several numerical examples have been done for different materials in the wedge and the half plane. The optimum shape of the wedge (including the bounding corner angle) is obtained, thus avoiding the stress singularity. The corner angle is compared to the critical wedge angle obtained from classical analysis. 


\section{Theoretical Background: Flat-Sided Wedge Contacting a Half Plane}

In Fig. 1, a half plane is compressed by a wedge with an interior angle $\gamma$. It is assumed that 1) the contacting bodies are isotropic and homogeneous, 2) the contact surface has a coefficient $\mu$ of Coulumb's dry friction, and 3) the deformation near the sharp wedge is well approximated by plane strain theory. The Airy stress function $\phi$ is introduced to satisfy the biharmonic equation (1). Stress [Eqs. (2-4)] and displacement [Eqs. 5 and 6] relations are given in polar coordinates:

$$
\begin{gathered}
\nabla^{4} \phi=0 \\
\sigma_{r r}=\frac{1}{r} \frac{\partial \phi}{\partial r}+\frac{1}{r^{2}} \frac{\partial^{2} \phi}{\partial \theta^{2}} \\
\sigma_{\theta \theta}=\frac{\partial^{2} \phi}{\partial r^{2}} \\
\sigma_{r \theta}=-\frac{1}{r} \frac{\partial^{2} \phi}{\partial r \partial \theta}+\frac{1}{r^{2}} \frac{\partial \phi}{\partial \theta} \\
\frac{\partial u_{r}}{\partial r}=\frac{1}{2 G}\left(\frac{1}{r} \frac{\partial \phi}{\partial r}+\frac{1}{r^{2}} \frac{\partial^{2} \phi}{\partial \theta^{2}}-\nu \nabla^{2} \phi\right) \\
r \frac{1}{r} \frac{\partial u_{r}}{\partial \theta}=\frac{1}{G}\left(-\frac{1}{r} \frac{\partial^{2} \phi}{\partial r \partial \theta}+\frac{1}{r^{2}} \frac{\partial \phi}{\partial \theta}\right)
\end{gathered}
$$

Boundary conditions are defined at the interface and free surfaces of the wedge and the half plane:

$$
\begin{gathered}
u_{\theta}^{\mathrm{I}}(r, 0)=u_{\theta}^{\mathrm{II}}(r, 0) \\
\sigma_{r \theta}^{\mathrm{I}}(r, 0)=\sigma_{r \theta}^{\mathrm{II}}(r, 0)=-\mu \sigma_{\theta \theta}(r, 0) \text { for } r>0 \\
\sigma_{\theta \theta}^{\mathrm{I}}(r, 0)=\sigma_{\theta \theta}^{\mathrm{II}}(r, 0) \\
\sigma_{r \theta}^{\mathrm{I}}(r,-\pi)=\sigma_{\theta \theta}^{\mathrm{I}}(r,-\pi)=0 \\
\sigma_{r \theta}^{\mathrm{II}}(r, \gamma)=\sigma_{\theta \theta}^{\mathrm{II}}(r, \gamma)=0
\end{gathered}
$$

where $\sigma_{\theta \theta}(r, 0)$ must be compressive and $u$ and $\sigma$ denote components of displacement and stress. The superscripts I and II refer to the half plane and wedge, respectively.

Several bounded-wedge problems were solved by Bogy to decide the order of the stress singularity. Bogy applied the Mellin transform to the boundary-value problems and obtained a general solution of the biharmonic equation (the Mellin transforms of Airy function). The general solution was used to obtain the Mellin transforms of the stresses, displacements, and boundary conditions. The eight system equations (the Mellin transforms of boundary conditions) involve eight unknown functions. The order of the stress singularity can be decided from the zeros of the determinant of the coefficients of the system equations. Algebraic details can be found in Refs. 5 and 6 .

If $p$ is a zero of $\mathcal{D}$ in $0<\operatorname{Re}(p) \leq 1$, the orders of the stress singularities may take the following forms:

$$
\sigma_{i j}=\left\{\begin{array}{c}
\mathcal{O}\left(r^{p-1}\right) \text { if } p \text { is real and } 0<p<1 \\
\mathcal{O}\left[r^{\xi-1} \cos (\eta \log r)\right] \text { or } \mathcal{O}\left[r^{\xi-1} \sin (\eta \log r)\right] \\
\quad \text { if } p=\xi+i \eta \text { is complex and } 0<\xi<1 \\
\mathcal{O}(\log r) \text { if } p=1 \text { and } \partial \mathcal{D} / \partial p=0 \text { at } p=1 \\
\mathcal{O}(1) \text { if there is no zero of } \mathcal{D} \text { in } \\
\quad 0<\operatorname{Re}(p)<1 \text { and } \partial \mathcal{D} / \partial p \neq 0 \text { at } p=1
\end{array}\right.
$$

Dundurs $^{22,23}$ shows that the influence of the elastic constants is governed by only two independent variables:

$$
\begin{gathered}
\alpha=\frac{\left(1-v_{1}\right) / G_{1}-\left(1-v_{2}\right) / G_{2}}{\left(1-v_{1}\right) / G_{1}+\left(1-v_{2}\right) / G_{2}} \\
\beta=\frac{1}{2} \frac{\left(1-2 v_{1}\right) / G_{1}-\left(1-2 v_{2}\right) / G_{2}}{\left(1-v_{1}\right) / G_{1}+\left(1-v_{2}\right) / G_{2}}
\end{gathered}
$$

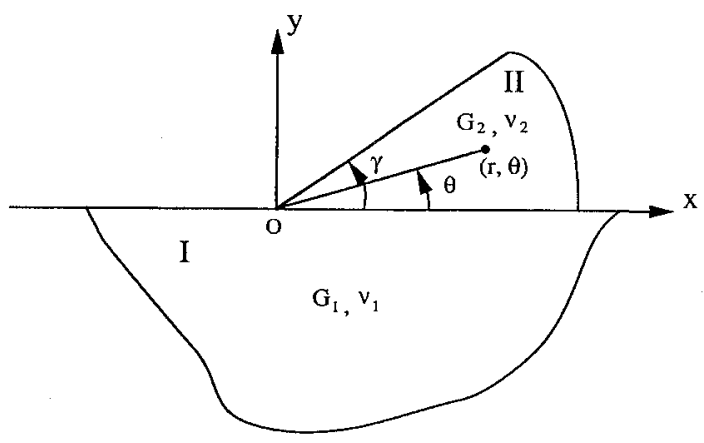

Fig. 1 Wedge in contact with a half plane.

The $\alpha$ is a measure of the difference in plane strain modulus ( 1 $\left.\nu^{2}\right) / E$. (In Ref. 8, Dundurs explains $\alpha$ as an index for the mismatch in the uniaxial compliance of the two bodies.) For special cases, $\alpha$ has different values: 1 for a rigid wedge and an elastic half plane, -1 for a rigid half plane and an elastic wedge, and 0 for an wedge and half plane with identical elastic materials.

Using the parameters $\alpha$ and $\beta$, one expresses the determinant of the coefficients of the system equations in rather simplified fashion:

$$
\mathcal{D}(p ; \gamma, \alpha, \beta, \mu)=8(1+p) \sin p \pi F(p ; \gamma, \alpha, \beta, \mu)
$$

where $\gamma$ is the wedge angle and

$$
\begin{aligned}
& F(p ; \gamma, \alpha, \beta, \mu)=(1+\alpha) \cos p \pi\left(\sin ^{2} p \gamma-p^{2} \sin ^{2} \gamma\right) \\
& \quad+\frac{1}{2}(1-\alpha) \sin p \pi(\sin 2 p \gamma+p \sin 2 \gamma)+\mu \sin p \pi \\
& \left.\quad \times\left[(1-\alpha) p(1+P) \sin ^{2} \gamma-2 \beta \sin ^{2} p \gamma-p^{2} \sin ^{2} \gamma\right)\right]
\end{aligned}
$$

The power singularities correspond to the real roots of $F(p ; \gamma, \alpha, \beta, \mu)$ in the interval $0<p<1$. In a study of the roots of $F(p ; \gamma, \alpha, \beta, \mu)$ for discrete values of physical parameters, Gdoutos has shown in Ref. 9 that there is at most one real root in the interval $0<p \leq 1$.

Comninou ${ }^{10}$ showed that no power singularities appear for certain combinations of $\gamma, \alpha, \beta$, and $\mu$ with $p=1$ and that the curve bounding the presence of power singularities is analytically expressed:

$$
\frac{\partial F(1 ; \gamma, \alpha, \beta, \mu)}{\partial p}=0
$$

Substitution of Eq. (16) into Eq. (17) leads to

$$
\alpha=\frac{(\pi+\gamma) \cos \gamma+(\pi \mu-1) \sin \gamma}{(\pi-\gamma) \cos \gamma+(\pi \mu+1) \sin \gamma}
$$

One can calculate the wedge angle $\gamma_{s}$ bounding power singularity for special cases of wedge contact. Based on Eq. (18), the bounding angle is shown in Fig. 2 for frictionless contact. A good geometric optimization method should move design points lying in the singular region into the nonsingular region.

\section{Geometric Strain Method for Shape Optimization}

The geometric strain method, developed by $\mathrm{Suh}^{20}$ and Suh et al., ${ }^{21}$ has not been widely published and will be reviewed here. A continuous body (Fig. 3) is considered for shape optimization. Surface tractions are applied on the boundary $\Gamma_{t}$. Body forces are applied in the domain $\Omega_{b}$. The boundary $\Gamma_{u}$ is constrained and the $\Gamma_{d}$ is an exterior design boundary, free to move. Here, $\Gamma_{i}$ is an interior boundary, which is fixed during the geometric strain calculation. The purpose of this study is to shape the exterior design boundary $\Gamma_{d}$ to satisfy a fully stressed state in a portion of $\Omega$ denoted by $\Omega_{f}$.

A typical approach to optimization is:

$$
\text { Minimize } V\left(x_{d}\right)=\int_{\Omega} \mathrm{d} v
$$




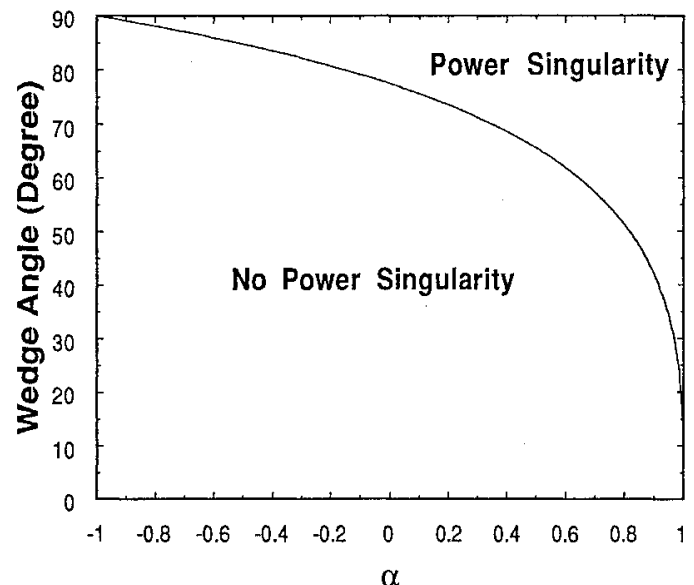

Fig. 2 Critical wedge angle for the presence of the power singularity.

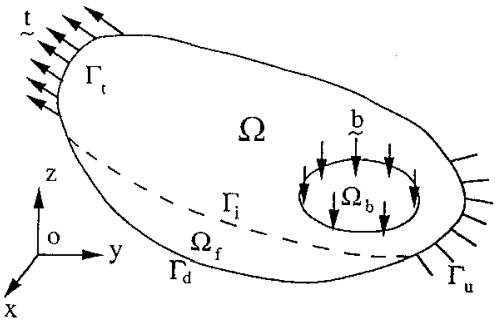

Fig. 3 Design domain.

Subject to

$$
f\left(\sigma_{1}(\boldsymbol{x}), \sigma_{2}(\boldsymbol{x}), \sigma_{3}(\boldsymbol{x})\right) \leq 0 \quad \forall \boldsymbol{x} \in \Omega_{f}
$$

where $x_{d}$ is a design variable vector that defines the shape of the exterior design boundary $\Gamma_{d} ; \sigma_{1}, \sigma_{2}$, and $\sigma_{3}$ are the components of principal stress; and functional $f$ represents a failure criterion, such as the maximum distortion energy theory.

We, however, will use an optimality criterion:

$$
f\left(\sigma_{1}(\boldsymbol{x}), \sigma_{2}(\boldsymbol{x}), \sigma_{3}(\boldsymbol{x})\right)=0 \quad \forall x \in \Omega_{f}
$$

where $\Omega_{f}$ is the fully stressed domain that satisfies the failure criterion. The GSM is derived from the optimality criterion equation (20) to obtain an optimum shape that satisfies the fully stressed design. Most practical solutions for fully stressed designs are either the optimum design or close to it. ${ }^{24}$

\section{Geometric Strain Method for Two-Dimensional Plane Problem}

The GSM is based on the stress ratio method, which is commonly used to attain the fully stressed design in one-dimensional truss problems. This idea is extended to obtain the fully stressed design of a general elastic body.

A two-dimensional infinitesimal element, as shown in Fig. 4, is introduced using the axes of the principal stresses at a point. Only normal stresses are considered in the principal coordinates.

The fully stressed state in the element can be obtained by changing the dimension of the perpendicular faces of the element. The amount of the length change that will lead to a fully stressed state is termed the geometric displacement.

It is assumed that the forces acting on the faces of the infinitesimal element remain constant at the values of $\sigma_{1} \Delta \ell_{2}$ and $\sigma_{2} \Delta \ell_{1}$. The current stresses can reach the objective stresses ( $\sigma_{o 1}$ and $\sigma_{o 2}$ in the principal axes of stress) by changing the length of the face of the element:

$$
\begin{aligned}
& \Delta \ell_{1}^{\prime}=\left|\sigma_{2} / \sigma_{o 2}\right| \Delta \ell_{1} \\
& \Delta \ell_{2}^{\prime}=\left|\sigma_{1} / \sigma_{o 1}\right| \Delta \ell_{2}
\end{aligned}
$$
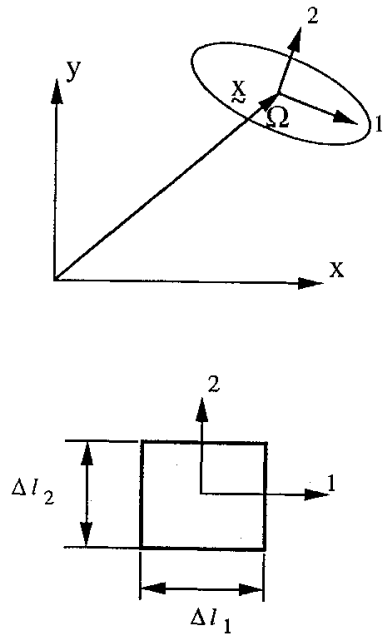

Fig. 4 Two-dimensional infinitesimal element in principal coordinates.

The principal geometric strain can be derived:

$$
\begin{aligned}
& \epsilon_{G 1}^{p}=\left(\Delta \ell_{1}^{\prime}-\Delta \ell_{1}\right) / \Delta \ell_{1}=\left|\sigma_{2} / \sigma_{o 2}\right|-1 \\
& \epsilon_{G 2}^{p}=\left(\Delta \ell_{2}^{\prime}-\Delta \ell_{2}\right) / \Delta \ell_{2}=\left|\sigma_{1} / \sigma_{o 1}\right|-1
\end{aligned}
$$

The geometric strain can be obtained from the principal geometric strain by transforming from the principal coordinates $(1,2)$ to the global coordinates $(x, y)$ :

$$
\left[\epsilon_{G}(x)\right]=[T(x)]^{T}\left[\epsilon_{G}^{p}(x)\right][T(x)]
$$

where $\left[\epsilon_{G}\right]$ is the matrix form of the geometric strain and $[T(x)]$ is the transformation matrix between the principal geometric strain and geometric strain in global coordinates. Note that $[T(x)]$ is the same as the transformation matrix between the principal stress and the stress in global coordinates:

$$
[T]=\left[\begin{array}{cc}
\cos \theta & \sin \theta \\
-\sin \theta & \cos \theta
\end{array}\right]
$$

The principal geometric strain $\left[\epsilon_{G}^{p}\right]$ is

$$
\left[\epsilon_{G}^{p}(\boldsymbol{x})\right]=\left[\begin{array}{cc}
\epsilon_{G 1}^{p}(x) & 0 \\
0 & \epsilon_{G 2}^{p}(x)
\end{array}\right]
$$

The geometric strain matrix is symmetric and can be represented in vector form:

$$
\epsilon_{G}=\left\{\begin{array}{c}
\epsilon_{G x} \\
\epsilon_{G y} \\
\gamma_{G x y}
\end{array}\right\}=\left\{\begin{array}{c}
{\left[\epsilon_{G}(1,1)\right]} \\
{\left[\epsilon_{G}(2,2)\right]} \\
2\left[\epsilon_{G}(1,2)\right]
\end{array}\right\}
$$

where $\epsilon_{G x}, \epsilon_{G y}$ are the $x$ and $y$ components of the normal geometric strains, respectively, and $\gamma_{G x y}$ is the shearing geometric strain. The geometric strain vector is defined in the fully stressed domain $\Omega_{f}$ and will be zero outside the domain.

The geometric displacement $u_{G}(x)$ is defined as the amount of the geometry change. The geometric displacement is obtained by converting the geometric strain into equivalent nodal loads and solving the load-displacement relation.

Since optimal shapes depend nonlinearly on design parameters, finding the optimal shape can require numerous iterations. In order to reduce the number of iterations, an acceleration factor is used. The product of the geometric displacement and the acceleration factor is added to the geometry of the current design to obtain a new design, which is expected to be the fully stressed design. The procedure is repeated after checking the stresses in the fully stressed domain $\Omega_{f}$ until convergence is achieved. 


\section{Shape Optimization of Contact Problems by Geometric Strain Method}

The GSM is divided into two phases. In the first phase, the stresses of a current design are obtained by the finite element method. In the second phase, the geometric strain is calculated from the stresses on the current design and a new design is found. The procedure is repeated until the response of the new design satisfies the optimality criterion.

First Phase: Finite Element Analysis

Use the finite element displacement formulation

$$
[K] \boldsymbol{u}=\boldsymbol{P}
$$

The displacement $\boldsymbol{u}$ is solved numerically from Eq. (29) and proper boundary conditions.

The stress $\sigma$ can be derived from the displacement $u$ :

$$
\boldsymbol{\sigma}=[E][D] \boldsymbol{u}
$$

where $[E]$ is the stress-strain matrix and $[D]$ is the straindisplacement matrix.

\section{Second Phase: Fully Stressed Design}

A new shape, closer to the fully stressed design, will be determined. The geometric strain is derived from the stresses in the first phase and from the optimality criterion. Geometric strain is defined at every Gauss point in the fully stressed domain $\Omega_{f}$. Similar to finite element analysis, the geometric strains are treated as if they were initial strains (due to thermal strains, say). The relation between equivalent and initial strains is well known. The initial strains are converted to these equivalent nodal loads. This becomes a new load case acting on the original system, but with different boundary conditions.

The geometric strain in element $e, \epsilon_{G}^{(e)}$, is interpolated by the geometric strains at all Gauss points of the element $e$ using the same displacement shape function used in the first phase:

$$
\epsilon_{G}^{(e)}(\boldsymbol{x})=\sum_{i=1}^{n_{e}} N_{i}(\boldsymbol{x}) \epsilon_{G}^{i}
$$

where $\boldsymbol{x}$ is the position vector with respect to the local coordinate of the element $e, N_{i}(x)$ is the displacement shape function, $\epsilon_{G}^{i}$ is the geometric strain at the Gauss point number $i$ of the element $e$, and $n_{e}$ is the total number of Gauss points in element $e$.

The geometric strains are treated as if they were initial strains in the finite element method and are converted to equivalent nodal loads. This becomes a new load case acting on the original system, but with different boundary conditions [Eq. (36)].

The equivalent nodal load $\boldsymbol{P}_{G}^{(e)}$ of element $e$ due to the geometric strain can be calculated:

$$
\boldsymbol{P}_{G}^{(e)}=\iiint_{V^{(e)}}[D(x)]^{T}[E]^{(e)} \epsilon_{\delta}^{(e)}(x) \mathrm{d} V
$$

Substitution of Eq. (31) into Eq. (32) leads to

$$
\boldsymbol{P}_{G}^{(e)}=\iiint_{V^{(e)}}[D(\boldsymbol{x})]^{T}[E]^{(e)}\left(\sum_{i=1}^{n_{e}} N_{i}(\boldsymbol{x}) \boldsymbol{\epsilon}_{G}^{i}\right) \mathrm{d} V
$$

The total equivalent load $\boldsymbol{P}_{G}$ is the summation of all the equivalent nodal loads of all elements:

$$
\boldsymbol{P}_{G}=\sum_{e=1}^{\mathrm{NE}} \boldsymbol{P}_{G}^{(e)}
$$

The stiffness matrix of the first phase, $[K]$ of Eq. (29), is used as the stiffness matrix for the equilibrium equation of the geometric displacement $\boldsymbol{u}_{G}$ :

$$
[K] \boldsymbol{u}_{G}=\boldsymbol{P}_{G}
$$

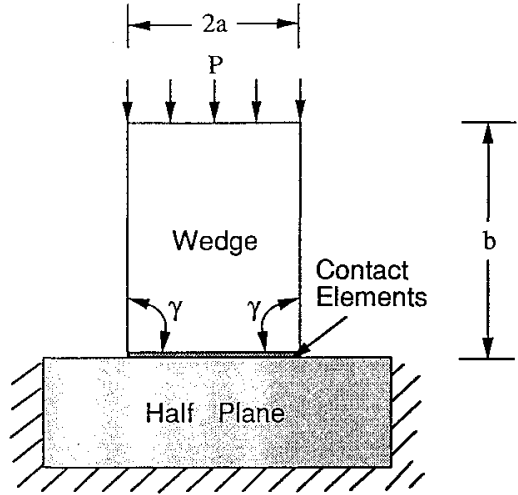

Fig. 5 Wedge in contact with a half plane.

The boundaries are held fixed except the exterior design boundary $\Gamma_{d}$ :

$$
\boldsymbol{u}_{G}(\boldsymbol{x})=0 \quad \forall \boldsymbol{x} \in\left(\Gamma-\Gamma_{d}+\Gamma_{i}\right)
$$

where $\Gamma$ is the boundary of the whole domain $\Omega$. The geometric displacement is the solution of Eqs. (35) and (36).

To obtain the new shape, every grid point on the design boundary $\Gamma_{d}$ is changed according to the geometric displacement obtained from Eqs. (35) and (36). Let $\boldsymbol{x}_{i}$ represent the position vector of the grid point $i$. Then the new position vector $x_{i}^{\text {new }}$ is

$$
\boldsymbol{x}_{i}^{\text {new }}=\boldsymbol{x}_{i}^{\text {old }}+\alpha_{f} \boldsymbol{u}_{G}^{i}
$$

where $\boldsymbol{u}_{G}^{i}$ is the geometric displacement vector of the grid point $i$ and $\alpha_{f}$ is an acceleration factor to speed convergence.

One replaces the old position vector of every grid point on the exterior design boundary $\Gamma_{d}$ with the new position vector Eq. (37) and keeps the current connectivity, which defines the relationship between element and grid points. Use of a single layer requires that the mesh be regenerated after each run. (Otherwise the strains in the outer layer would become very large). The finite element model of the new shape can be automatically obtained unless there is a severely distorted element. If the design boundary is severely wrinkled or the elements are severely distorted, a smoothing technique for the design boundary is used and the elements for the whole domain $\Omega$ are remeshed.

\section{Numerical Examples}

As considered in the theory of a wedge contact, the stress singularity may disappear at certain combinations of parameters $\alpha, \beta$ and wedge angle $\gamma$. The present numerical study investigates whether the stress singularity can be avoided by changing the shape of a wedge by using the GSM. A local wedge angle at the contact point is obtained as a part of the optimum shape, and the stress singularity disappears.

Numerical examples for a wedge contacting a half plane (Fig. 5) use various materials in the wedge and the half plane. The optimal wedge shape is sought. A fixed load $P$ is uniformly distributed on the top of the wedge. The distance $2 a$ is held fixed during the optimization. The method tends to minimize volume of material under a fixed load.

Results will be presented in the form of figures of baseline and optimal shapes. Stresses in the baseline and optimum designs will be presented to recognize the presence/absence of the stress singularity. Finally, the optimal wedge angles from the numerical method and critical wedge angles from the analytical method will be compared.

\section{Baseline Design Model}

A half model is used for the study by using reflective symmetry (Fig. 6) for reduction of size problem. Dimensions are $a=40 \mathrm{~mm}$ and $b=80 \mathrm{~mm}$. The initial shape of the design boundary is straight but is subsequently allowed to curve. For the finite element model in Fig. 7, 182 nodes and 153 elements are used. The wedge has 96 elements and the half plane has 48 elements. Four-node plane-strain quadrilateral elements are used. 


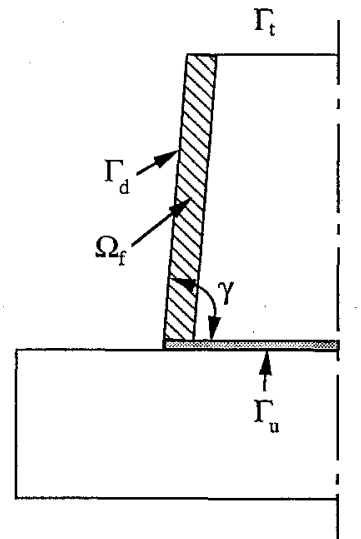

Fig. 6 A half of the wedge in contact with a half plane.

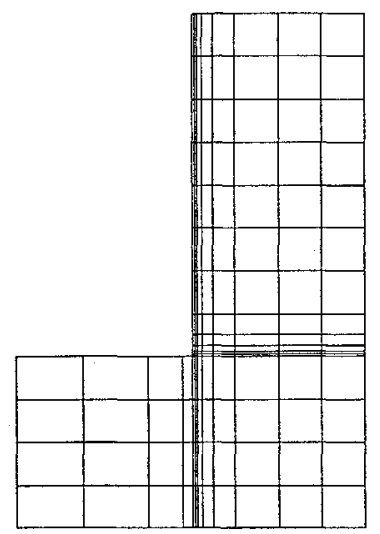

Fig. 7 Baseline design of wedge in contact with a half plane.

The wedge and the half plane are connected by nine frictionless, linear contact elements. The contact elements transfer only compression, have no length, and are sized to carry equally distributed force. The stiffnesses of the contact elements are very high $\left(10^{6}\right.$ greater than stiffness of the wedge and the half plane). The distributed load $P=300 \mathrm{~N} / \mathrm{mm}$. The bottom, left, and right sides of the half plane are clamped.

The purpose of the present study is to avoid a stress singularity rather than to find exact stress fields near the sharp edge. Therefore, extremely fine finite meshes, usually used in the numerical analysis of stress singularities, are not used. However, somewhat fine meshes (on the order of a half millimeter) are required to model high stresses around the sharp edge, as shown in Fig. 7.

For design optimization by the GSM, 13 design nodes and 13 stress constraints are used. The design region $\Omega_{f}$ is the single outer layer of wedge elements. The stress constraint is that maximum principal stress in this region should not exceed $110 \%$ of the applied stress. The design boundary in this problem can be moved horizontally, except for the top node, which is fixed. All boundaries of the whole domain $\Omega$, except the exterior design boundary $\Gamma_{d}$, are fixed. The constrained domain is given fixed boundary conditions in the solution procedure of the geometric displacement-equivalent load. If all stresses in the design boundary are less than $110 \%$ of the applied stress $(300 \mathrm{MPa})$, then it is assumed that the stress singularities along the sharp edge have disappeared. The $110 \%$ number is indeed arbitrary. If a looser criterion is used, the solution converges more quickly, to an angle $\gamma$ that is farther from the boundary (between singular and nonsingular behavior). If a tighter criterion is used (105\%, say), then the solution takes longer to converge and gives an angle tighter to the singular limit. By starting with a feasible (nonsingular) solution and using a tight stress constraint, optimum designs reach conservative shapes that are close to the optimum on the feasible (nonsingular) side.

The effect of mesh refinement has not been studied because of the labor involved in automated remeshing at different scales. The

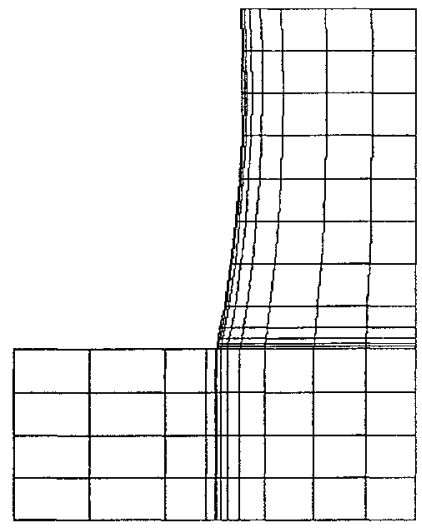

Fig. 8 Optimal wedge shape for the wedge and the half plane (identical materials)

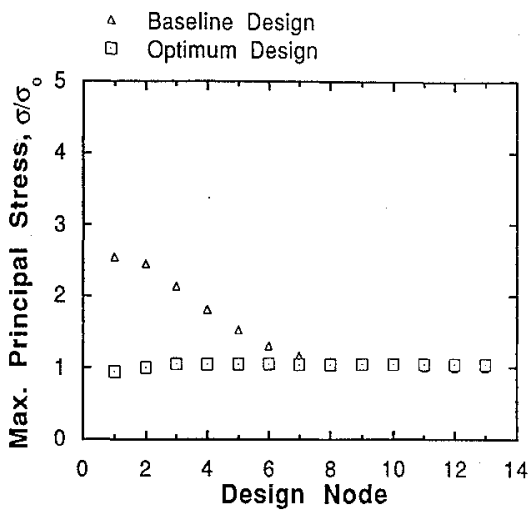

Fig.9 Maximum principal stresses at the baseline and optimum design (identical materials)

mesh used provides accuracy of the critical angle within $1.7 \mathrm{deg}$ in all cases. A finer mesh would provide even closer correlation with the analytical solution.

\section{Example 1: Wedge Contacting Half Plane with Identical Materials (Frictionless)}

Starting with the baseline design in Fig. 7, the GSM obtains the shape in Fig. 8 after eight iterations. The wedge angle at the contact point is $78.8 \mathrm{deg}$. Maximum principal stresses are shown in Fig. 9 for the baseline and optimum designs. The singularity that exists in the initial baseline design disappears in the optimum design.

The critical wedge angle from contact mechanics can be obtained from Eq. (18) for identical elastic materials $(\alpha=0)$ and smooth contact $(\mu=0)$ :

$$
-(\pi+\gamma)+\tan \gamma=0
$$

yielding $\gamma_{s}=77.45 \mathrm{deg}$.

The optimum wedge angle $\gamma=78.8 \mathrm{deg}$ by the GSM is $1.7 \%$ higher than the critical wedge angle $\gamma_{s}=77.45 \mathrm{deg}$, placing the design slightly within the "dangerous" region of Fig. 2. The GSM result therefore needs to have some design conservatism added, perhaps by "sharpening" the contact angle by $2 \mathrm{deg}$.

\section{Example 2: Wedge Contacting Half Plane with Different Elastic Materials $\left(G_{1}=2 G_{2}\right.$ and $\left.\nu_{1}=\nu_{2}=0.3, \alpha=-\frac{1}{3}\right)$}

In this example, the material of the half plane is twice as stiff as the wedge. After five iterations, starting from the baseline design (Fig. 7), the GSM provides the optimum wedge angle $\gamma=83.7$ deg. The optimal shape of the wedge is shown in Fig. 10. Maximum principal stresses are shown in Fig. 11 for the baseline and optimum designs. The singularity that exists in the initial baseline design again disappears in the optimum design.

The critical wedge from contact mechanics can be obtained from Eq. (18) for the doubly stiff half plane $G_{1}=2 G_{2}$ and $v_{1}=v_{2}=$ 


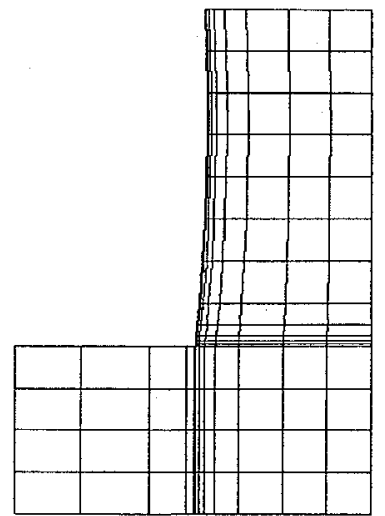

Fig. 10 Optimal wedge shape for $G_{1}=2 G_{2}$ and $\nu_{1}=\nu_{2}=0.3$.

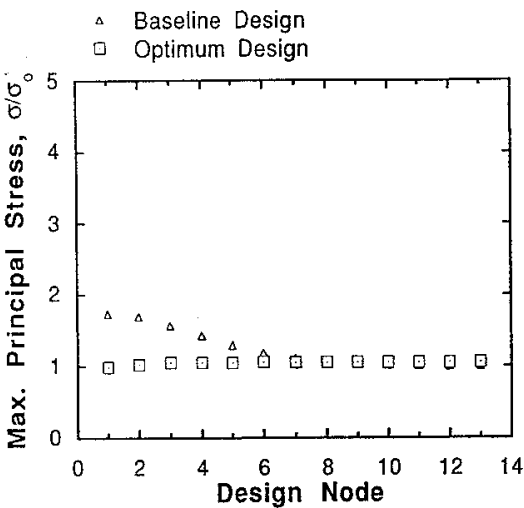

Fig. 11 Maximum principal stresses at the baseline and optimum design with $G_{1}=2 G_{2}$ and $\nu_{1}=\nu_{2}=0.3$.

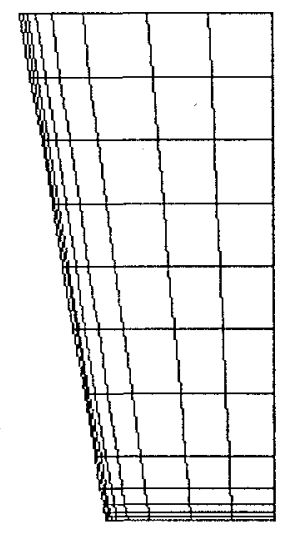

Fig. 12 Baseline design of elastic wedge in contact with a rigid half plane.

$0.3\left(\alpha=-\frac{1}{3}\right)$ and frictionless contact $(\mu=0)$ :

$$
2 \pi+\gamma-\tan \gamma=0
$$

yielding $\gamma_{s}=82.62 \mathrm{deg}$.

The optimum wedge angle $\gamma=83.7 \mathrm{deg}$ by the GSM is $1.3 \%$ higher than the critical wedge angle $\gamma_{s}=82.62 \mathrm{deg}$.

\section{Example 3: Elastic Wedge Contacting Rigid Half Plane} $\left(G_{1}=\infty, \alpha=-1\right)$

This case belongs to the Signorini problem, which is a contact problem of a linearly elastic body with a rigid frictionless foundation. For modeling this problem, only the wedge is modeled. The bottom surface of the wedge is vertically constrained and horizontally free. The wedge angle for the baseline (Fig. 12) is chosen to be $105 \mathrm{deg}$, different from the $90 \mathrm{deg}$ rectangle in the previous examples in order to start with an infeasible design. (The exact solution is the vertical edge.)

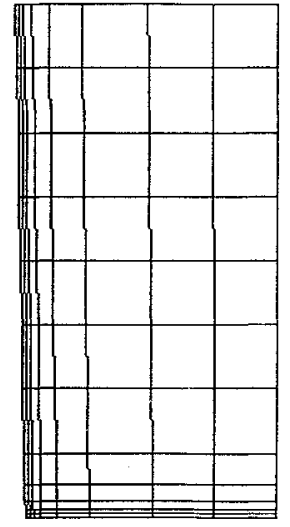

Fig. 13 Optimum shape of elastic wedge in contact with a rigid half plane.

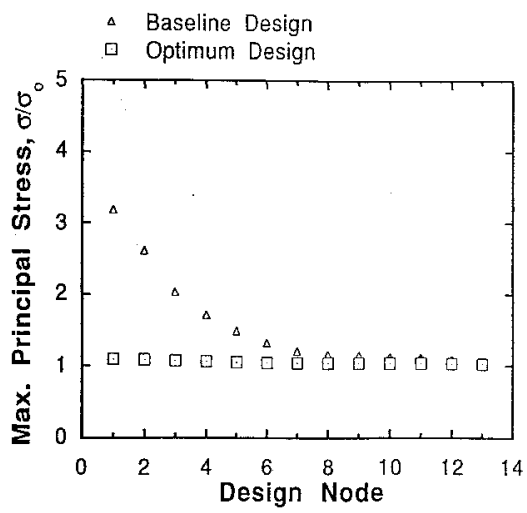

Fig. 14 Maximum principal stresses at the baseline and optimum design for an elastic wedge and a rigid half plane problem.

The optimal wedge angle $\gamma=91.3 \mathrm{deg}$ is obtained by the GSM after six iterations from the baseline design. The optimal wedge shape (Fig. 13) has the maximum principal stresses in Fig. 14 for the baseline and optimum designs. Again, the singularity that exists in the initial baseline design disappears in the optimum design.

For a comparison with contact analysis, set $\alpha=-1$ in Eq. (18)

$$
1+\mu \tan \gamma=0
$$

which yields $\gamma_{s}=90 \mathrm{deg}$ for frictionless contact $(\mu=0)$. The optimal wedge angle $\gamma=91.3 \mathrm{deg}$ is $1.5 \%$ higher than the critical wedge angle $\gamma_{s}=90 \mathrm{deg}$ from the theory of contact mechanics.

\section{Conclusions}

A numerical method has been devised to optimize the shape of solid bodies in the presence of stress singularities. The numerical tools used are finite element analysis and the GSM. Numerical examples of a plane-strain wedge contacting a half plane were solved for frictionless contact involving identical materials, stiffer wedge, stiffer half plane, and rigid half plane.

It was proven that the numerical approach is useful to reduce the high stress in the sharp-wedge contact problem. From the design standpoint, it would be wise to "sharpen" the contact angle (make the included angle more acute) by 2 deg to account for the approximation of such a coarse mesh. A better alternative would be to refine the mesh.

The numerical approach can be extended to more general contact problems such as frictional contact and contact between threedimensional bodies. Work is underway in this direction.

\section{Acknowledgment}

Special thanks are given to Anthony M. Waas, The University of Michigan, who noticed that the optimal shape of a geometric strain method solution avoided a stress singularity in the case of a valve-spring retainer in Ref. 21. 


\section{References}

${ }^{1}$ Timoshenko, S. P., History of Strength of Materials, Dover, New York 1952.

${ }^{2}$ Timoshenko, S. P, and Goodier, J. N., Theory of Elasticity, McGrawHill, New York, 1970.

${ }^{3}$ Barber, J. R., Elasticity, Kluwer Academic, Norwell, MA, 1992.

${ }^{4}$ Williams, M. L., "Stress Singularities Resulting from Various Boundary Conditions in Angular Corners of Plates in Extension," Journal of Applied Mechanics, Vol. 19, Dec. 1952, pp. 526-528.

${ }^{5}$ Bogy, D. B., "Two Edge-Bonded Elastic Wedges of Different Materials and Wedge Angles under Surface Tractions," Journal of Applied Mechanics, Vol. 38, June 1971, pp. 377-386.

${ }^{6}$ Bogy, D. B., "On the Problem of Edge-Bonded Elastic Quarter-Planes Loaded at the Boundary," International Journal of Solids and Structures, Vol. 6, 1970, pp. 1287-1313.

${ }^{7}$ Muskhelishvili, N. I., Some Basic Problems of the Mathematical Theory of Elasticity, translated from the Russian by J. R. M. Radok, Noordhoof, Groningen, Netherlands, 1963.

${ }^{8}$ Dundurs, J., and Lee, M. S., "Stress Concentration at a Sharp Edge in Contact Problems," Journal of Elasticity, Vol. 2, No. 2, 1972, pp. 109-112.

${ }^{9}$ Gdoutos, E. E., and Theocaris, P. S., "Stress Concentrations at the Apex of a Plane Indenter Acting on an Elastic Half Plane," Journal of Applied Mechanics, Vol. 42, Sept. 1975, pp. 688-692.

${ }^{10}$ Comninou, M., "Stress Singularity at a Sharp Edge in Contact Problems with Friction," Journal of Applied Mathematics and Physics (ZAMP), Vol. 27, 1979, pp. 493-499.

${ }^{11}$ Peterson, R. E., Stress Concentration Factors, Wiley, New York, 1974.

${ }^{12}$ Bijak-Zochowski, M., Waas, A. M., Anderson, W. J., and Miniatt, C. E. "Reduction of Contact Stress by Use of Relief Notches," Experimental Mechanics, Vol. 31, Sept. 1991, pp. 271-275.

${ }^{13}$ Benedict, R. L., and Taylor, J. E., "Optimal Design for Elastic Bodies in Contact," Proceedings of NATO-ASI, Optimization of Distributed Parameter
Structures, edited by E. J. Haug and J. Cea, Vol. 2, Sijthoff and Noordhoff, Netherlands, 1981, pp. 1553-1599.

${ }^{14}$ Benedict, R. L., "Optimal Design for Elastic Bodies in Contact," Ph.D. Dissertation, University of Michigan, Ann Arbor, MI, 1980.

${ }^{15}$ Taylor, J. E., "Distributed Parameter Optimal Structural Design: Some Basic Problem Formulations and Their Applications," Proceedings of NATO. ASI, Computer Aided Optimal Design: Structural and Mechanical Systems, edited by C. A. M. Soares, Springer-Verlag, Berlin, 1981, pp. 3-85.

${ }^{16}$ Haslinger, J., and Neittaanmäki, P., Finite Element Approximation for Optimal Shape Design: Theory and Applications, Wiley, New York, 1988.

${ }^{17}$ Haslinger, J., "Contact Shape Optimization: The Mathematical Theory," Proceedings of Contact Mechanics: International Symposium, Nov. 1992, pp. 287-303.

${ }^{18}$ Feijóo, R. A., and Francello, E. A., "A Finite Element Approach for an Optimal Shape Design in Contact Problems," Proceedings of Contact Mechanics: International Symposium, Nov. 1992, pp. 263-286.

${ }^{19}$ Kikuchi, N., and Oden, J. T., Contact Problems in Elasticity: A Study of Variational Inequalities and Finite Element Methods, Society of Industrial and Applied Mathematics, Philadelphia, 1988.

${ }^{20}$ Suh, M. W., "Geometric Strain Method for Shape Optimization," Ph.D. Dissertation, University of Michigan, Ann Arbor, MI, 1989.

${ }^{21}$ Suh, M. W., Anderson, W. J., and McDonald, J. P., "Application of Geometric Strain Method to Shape Optimization of Spring Retainer Redesign," Computer Aided Optimum Design of Structures: Applications, OPTI-89, Southampton, United Kingdom, June 1989, pp. 189-198.

${ }^{22}$ Dundurs, J., "Discussion," Journal of Applied Mechanics, Sept. 1969, pp. 650-652.

${ }^{23}$ Dundurs, J., "Effect of Elastic Constant on Stress in a Composite Under Plane Deformation," Journal of Composite Material, Vol. 1, 1967, pp. 310322.

${ }^{24}$ Kirsch, U., Optimum Structural Design, McGraw-Hill, New York, 1981. 